CIC. Cuadernos de Información y Comunicación ISSN: 1135-7791

http://dx.doi.org/10.5209/CIYC.55965

\title{
Efectos de las bases de conocimiento hipertextuales semánticamente estructuradas en las estructuras de conocimiento de los usuarios ${ }^{1}$
}

\author{
David H. Jonassen ${ }^{2}$
}

Propuesto: 12 de diciembre de 2016 / Aceptado: 13 de diciembre de 2016.

\section{In Memoriam Prof. David Jonassen (1946-2012)}

Resumen. Algunos investigadores y diseñadores de hipertextos creen que las estructuras de información de los hipertextos deberían reflejar las estructuras de la memoria humana y que, al derivar empíricamente y cartografiar la estructura semántica de la información sobre el hipertexto, y al ilustrar explícitamente esa estructura en la interfaz hipertextual, se efectuarán mayores cambios en las estructuras de conocimiento de los usuarios (Jonassen, 1990, 1991b; Lambiotte et al., 1989; McAleese, 1990; McDonald, Paap y McDonald, 1990). Este capítulo presenta técnicas para averiguar la estructura del conocimiento de un experto y organizarla en un hipertexto. Después, se analizarán los resultados de una serie de estudios en curso que comprueban dichas ideas. Estos estudios muestran que el mero hecho de representar las estructuras de contenido en la interfaz no es suficiente para ayudar a los estudiantes a adquirir dicha estructura. Son la naturaleza de la tarea de procesamiento y los objetivos de aprendizaje al interactuar con un hipertexto los que en realidad determinan los efectos de su uso en las estructuras de conocimiento de los estudiantes.

Palabras clave: hipertexto; carga cognitiva; arquitectura informacional; educación; tipos de carga cognitiva.

\section{[en] Effects of semantically structured hypertextual knowledge bases on user knowledge structures}

Abstract. Some hypertext researchers and designers believe that hypertext information structures should reflect the structures of human memory and that by empirically deriving and then mapping the semantic structure of information onto hypertext and explicitly illustrating that structure in the hypertext interface will result in greater changes in the knowledge structures of the users (Jonassen, 1990, 1991b; Lambiotte et al., 1989; McAleese, 1990; McDonald, Paap and McDonald, 1990). This chapter introduces techniques for ascertaining an expert's knowledge structure and mapping it onto hypertext. It then reviews the results of an ongoing series of studies that test these ideas. The studies show that merely illustrating content structures in the interface is not sufficient for helping learners acquire that

Traducción de Daniel Alonso Martínez.

2 David Jonassen fue profesor en las Universidades de Missouri y Colorado, investigador en educación y lenguajes en las nuevas tecnologías, autor de centenares de artículos y capítulos y decenas de textos de investigación sobre las innovaciones cognitivas y los diseños educativos necesarios en los nuevos entornos digitales. Profesor laureado y premiado en múltiples universidades del mundo, donde ejerció docencia, en 28 países de los continentes de Australia, Europa, America y Asia, falleció en 2012. Sus aportaciones en el análisis de las arquitecturas informacionales y carga cognitiva en los nuevos hipertextos digitales ha sido pionera y ha enlazado con los estudios de la semántica y sintaxis discursiva, así como con los estudios educacionales en el cambio tecnológico digital. 
structure. Rather, it is the nature of the processing task and goals for learning whilst interacting with a hypertext that determines the effects of its use on learners' knowledge structures.

Keywords: hypertext; cognitive load; informational design; instructional design; tupes of cognitive load.

Cómo citar: Jonassen, D. H. (2017). Efectos de las bases de conocimiento hipertextuales semánticamente estructuradas en las estructuras de conocimiento de los usuarios, en CIC. Cuadernos de Información y Comunicación 22, 47-62.

\section{Redes Asociativas}

Algunos investigadores han afirmado que el hipertexto imita las redes asociativas de la memoria humana (Fiderio 1988; Jonassen, 1990, 1991a, 1991b; McAleese, 1990; McDonald, Paap y McDonald, 1990). La teoría de los esquemas y las redes estructurales activas han proporcionado las bases fundamentales principales para el desarrollo de hipertextos asociativamente estructurados (Jonassen, 1989b). La teoría de los esquemas (Rumelhart y Orton, 1977) afirma que el conocimiento es almacenado en paquetes de información o esquemas que contienen nuestros constructos mentales para las ideas. Los esquemas tienen atributos, los cuales en su mayoría consisten en otros esquemas. Cada esquema que construimos representa un mini-marco en el que interrelacionar información sobre un tema en una única unidad conceptual. Estos esquemas están organizados en una red de conceptos interrelacionados conocida como red semántica. Dichas estructuras en red se componen de nodos o ideas y las relaciones descritas que las conectan (Norman, Gentner y Stevens, 1976). Los nodos son representaciones de conceptos o esquemas, y los enlaces definen las relaciones proposicionales entre ellas. Las estructuras semánticas en el hipertexto representan los esquemas como nodos y las relaciones entre esquemas como enlaces de hipertexto. La razón para hacerlo así es que al cartografiar explícitamente la red semántica de un experto sobre un hipertexto, los estudiantes podrían llegar a pensar como un experto más fácilmente. Estas motivaciones se explicarán con mayor profundidad en secciones subsiguientes.

\section{Motor asociativo del hipertexto}

Bush (1945) observó que las ideas surgen de "la asociación de pensamientos, en consonancia con una intrincada red de rastros dejados por células del cerebro". Esta observación, junto con el continuo desarrollo de la teoría de la red asociativa, han provisto de una base conceptual para el desarrollo de los hipertextos. El hipertexto, al igual que otras tecnologías como las bases de datos y los sistemas expertos, es un sistema basado en el conocimiento. Esto significa que el contenido sobre un tema es almacenado en una base de conocimiento estructurada por un modelo de datos particular. El modelo de datos define la organización de la información. Esta organización a su vez define las relaciones entre las informaciones en la base de conocimiento. La lógica en cada tipo de modelo de datos varía en función de las clases de relaciones que lo componen. La lógica derivada del modelo de datos proporciona un motor para el acceso y uso de la información de la base de conocimiento. La mayoría de los expertos en sistemas, por ejemplo, utilizan un motor basado en reglas, las cuales expresan relaciones entre con- 
diciones y consecuencias (p. ej., IF velocidad $<$ V1 AND posición en pista $>7$ AND potencia $=>95 \%$, THEN abortar despegue).

Por otro lado, un motor de hipertexto es asociativo. Esto significa que se basa en una red asociativa de ideas. Un modelo de datos asociativo, debido a la cantidad de relaciones posibles, no constriñe las relaciones o enlaces tanto como un motor basado en reglas. Los enlaces pueden definirse mediante un conjunto de asociaciones abiertas, de modo que las estructuras de los hipertextos pueden tomar una variedad de formas. Por lo tanto, las estructuras del hipertexto son maleables y la estructura lógica de cualquier hipertexto podría emular las estructuras lógicas de numerosos diseños o funciones instructivos (Jonassen, 1991b). Si el hipertexto imita las redes asociativas humanas, entonces el motor hipertextual debería reflejar la red semántica de un intérprete o experto experimentado. Esto significa que podemos cartografiar la estructura del conocimiento asociativo de un experto o erudito y plasmarla en la estructura de un hipertexto. En el próximo apartado, explicaré cómo los hipertextos pueden emular las estructuras de conocimiento experto y cómo valorar los cambios en las estructuras de conocimiento de los aprendices como resultado de la interacción con dichos hipertextos.

\section{Cartografiando el conocimiento en un hipertexto}

\section{El aprendizaje y las redes semánticas}

Como se ha señalado más arriba, nuestras redes semánticas son representaciones conceptuales de lo que somos. El conocimiento interrelacionado dentro de la memoria semántica permite a los humanos combinar ideas, inferir, extrapolar o cualquier otra clase de razonamiento con la información. Aprender consiste en construir nuevas estructuras asimilando información del entorno y erigiendo nuevos nodos que los describan y se interrelacionen con otros nodos existentes y entre ellos mismos (Norman, 1976). Esto requiere la formación de enlaces entre el conocimiento existente y el nuevo conocimiento para comprender la información del entorno. Por tanto, el aprendizaje podría concebirse como una reorganización de las estructuras de conocimiento del estudiante resultante de las interacciones del mismo con su entorno. Esta hipótesis ha sido demostrada empíricamente. A consecuencia de la enseñanza, la estructura de conocimiento del alumno es muy parecida a la del instructor (Shavelson, 1974; Thro, 1978). De acuerdo a esta conceptualización, aprender es cartografiar el conocimiento de un tema (habitualmente aquél detentado por el profesor o experto) sobre la estructura de conocimiento de un estudiante.

Los motores o estructuras de los hipertextos podrían diseñarse para reflejar la estructura semántica de un experto en alguna materia. La pregunta de investigación implícita en estas suposiciones es, "si la estructura de nodos y enlaces del hipertexto refleja la estructura semántica del experto, ¿Será más eficientemente plasmada la estructura de conocimiento del experto sobre el navegador ${ }^{3}$ del aprendiz?" Esta es la pregunta que se ha investigado en los estudios descritos a continuación.

Investigar esta cuestión requiere la definición de nuevos métodos y herramientas de estudio. Si aceptamos la premisa anteriormente expuesta, que aprender es re-

(N. del T.) Se traduce aquí el término "browser" como "navegador". 
organizar las estructuras de conocimiento, entonces necesitamos herramientas para describir las representaciones del conocimiento y exponerlas durante el aprendizaje. Si queremos diseñar hipertextos que reflejen la estructura semántica de un experto, entonces necesitamos medios para evaluar la estructura de conocimiento del experto y después transmitirla al estudiante. Jonassen (1990) revisó tres métodos para la evaluación de las estructuras de conocimiento. El mejor y más extendido de dichos métodos es la graduación de listas de libre asociación de palabras (Preece, 1976). Cuando se proporciona una lista vacía, el alumno genera una lista de asociaciones libres a esa palabra. Las palabras que se recuerden primero son las más fuertemente asociadas. Se puede calcular un mapa semántico (un mapa espacial que refleja las distancias semánticas entre ideas) a partir de estas asociaciones calculando coeficientes de relación y escalando multidimensionalmente dichos coeficientes (Jonassen, 1987; Shavelson, 1974) lo que produce un mapa n-dimensional que ilustra las asociaciones semánticas en un ámbito de contenido. Este mapa se convierte en la interfaz de navegación.

Otro método más simple para cartografiar estructuras de conocimiento es una técnica de notación conocida como notación de patrones (Buzan, 1974; Fields, 1982). El anotador de patrones comienza apuntando la idea central en el centro de una página y usando una técnica de libre asociación para añadir conceptos relacionados que irradian de dichas líneas para crear un mapa del ámbito de contenido. Este método ha demostrado que replica de forma precisa las mismas estructuras que aquellas producidas por la libre asociación de palabras (Jonassen, 1987).

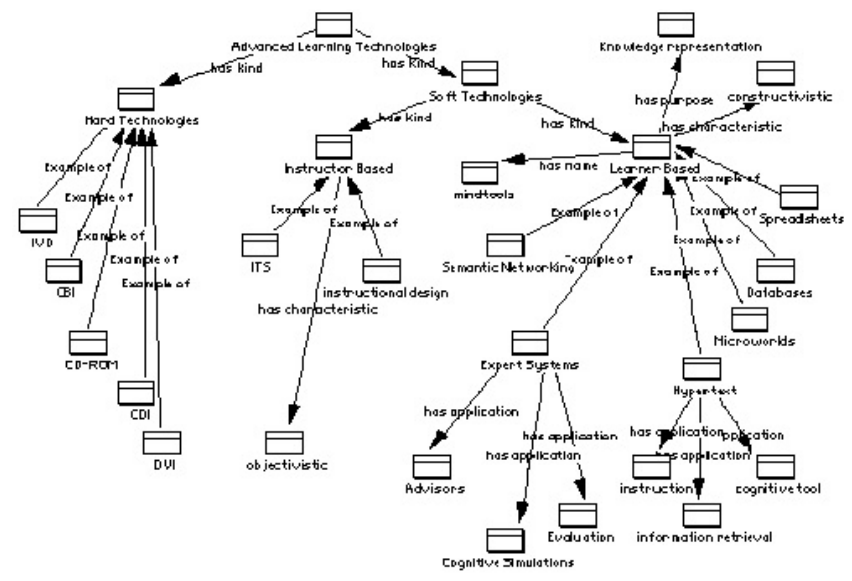

Figura 1. Parte de un mapa semántico producido con Learning Tool

Los mapas semánticos producidos tanto por la asociación de palabras como por la notación de patrones han sido implementados en entornos computacionales. Programas como Learning Tool (Kozma, 1987), SemNet (Fisher et al., 1988), y Pathfinder Nets (McDonald, Paap y McDonald, 1990) facilitan la producción de mapas semánticos. La figura 1 ilustra parte de un mapa semántico producido con Learning Tool. Los esudiantes podrían utilizar este programa como estrategia cognitiva para aumentar el valor significativo de la información y favorecer la adquisición de conocimientos. El programa podría también utilizarse para evaluar los efectos de reorganización del conocimiento propios del aprendizaje. 


\section{Redes semánticas para diseñar hipertextos}

Si aceptamos la sugerencia de que el hipertexto debería reflejar la estructura semántica de un intérprete o experto experimentado, entonces necesitamos métodos para plasmar esas redes de estructuras semánticas en el hipertexto. Esto podría realizarse haciendo que un experto o un grupo de individuos experimentados o expertos generaran mapas semánticos utilizando cualquiera de las técnicas descritas más arriba. Los mapas proporcionan una estructura de enlaces y nodos que podrían ser directamente plasmados en la interfaz hipertextual. Los conceptos en los mapas semánticos comprenden los nodos hipertextuales y las relaciones son, de hecho, enlaces de hipertexto. La capacidad de trabajar de forma interactiva repetidamente con un experto o grupo de individuos versados para definir, clarificar, y corregir estas estructuras debería proporcionar mapas más significativos y provechosos. La adopción de este método, el uso de mapas semánticos para definir el modelo estructural de un hipertexto mediante la elaboración directa de mapas de la organización de ideas en hipertextos, ha sido objeto de un debate considerable (Jonassen y Mandl, 1990) y es el tema del conjunto de estudios descritos más adelante.

\section{Desarrollo de navegadores gráficos a partir de mapas semánticos}

El camino más directo para cartografiar la estructura semántica del experto sobre un hipertexto es utilizar el mapa semántico como un navegador gráfico en el hipertexto. Los navegadores gráficos son mapas o listados gráficos de los nodos disponibles en un hipertexto. Representan una interfaz gráfica entre el usuario y el hipertexto, diseñado para reducir los problemas de navegación dentro del hipertexto (Jonassen, 1988). Perderse en una gran red de nodos hipertextuales y enlaces es un problema habitual entre los usuarios de hipertextos, por lo que los navegadores gráficos se diseñan para proporcionar un mapa espacial de la organización de los nodos en un hipertexto. A menudo, sin embargo, la disposición o estructura de los nodos que se muestran en un navegador gráfico es arbitraria (p. ej., filas y columnas de nodos). La hipótesis de nuestra investigación reciente (Jonassen y Wang, 1990; 1991) es que al disponer los nodos en un navegador gráfico según el mapa semántico de un experto, se transmite de forma explícita la organización de ideas en la estructura de conocimiento del experto. Es decir, se muestra al usuario cómo piensa el experto. Por lo tanto, al navegar a través de un hipertexto, el usuario está de hecho navegando por la estructura de conocimiento del experto. Las preguntas de investigación que hemos desarrollado se centran en la medida en que la estructura semántica mostrada en los navegadores gráficos verdaderamente se traza en la estructura de conocimiento del usuario. ¿Hasta qué punto el usuario forma o replica esa estructura en sus propias representaciones del conocimiento?

\section{Investigación}

Hemos adoptado una serie de estudios empíricos para evaluar los efectos de la estructuración semántica explícita del hipertexto en las estructuras de conocimiento de 
los alumnos. Queríamos saber hasta qué medida un hipertexto semánticamente estructurado afecta a la adquisición de conocimiento ordenado. Describiré brevemente la metodología y los métodos de evaluación comunes a todas las investigaciones y después repasaré los métodos y resultados específicos de cada estudio, a continuación analizaré las conclusiones e implicaciones de esta investigación.

\section{Métodos}

A fin de valorar los efectos del hipertexto semánticamente estructurado en las estructuras de conocimiento de los estudiantes, era necesario desarrollar instrumentos que evaluaran el conocimiento estructural de los estudiantes. El conocimiento estructural es el conocimiento de cómo los conceptos dentro de un dominio se interrelacionan (Diekhoff, 1983). El conocimiento estructural permite a los estudiantes formar las conexiones necesarias para describir y utilizar guiones o esquemas complejos. Es una forma de conocimiento conceptual que media la traducción de conocimiento declarativo a conocimiento procedimental. El conocimiento procedimental se basa en el conocimiento estructural para efectuar las relaciones funcionales entre las ideas.

A fin de valorar la adquisición de conocimiento estructural siguiendo varios tratamientos, hemos desarrollado tres subescalas de diez preguntas, cada una para medir distintos aspectos del conocimiento estructural: a) juicios de relación de proximidad, b) relaciones semánticas, y c) analogías. Todas las preguntas del cuestionario sobre conocimiento estructural fueron desarrolladas para centrarse en las relaciones entre conceptos de importancia contenidos en el hipertexto. Los juicios de relación de proximidad requerían que los estudiantes asignaran un número entre el $1 \mathrm{y}$ el $19 \mathrm{a}$ cada uno de los diversos pares de conceptos para indicar cómo de fuerte era la relación que, según ellos pensaban, existía entre los conceptos de cada par (Diekhoff, 1983). Por ejemplo:

- recuperación de información y documentación online

- procesamiento de hipertexto y base de datos

Las subescala de relaciones semánticas constaban de preguntas de selección múltiple que pedían a los estudiantes identificar la naturaleza de las relaciones entre dos conceptos. Estas relaciones eran tomadas de la base de conocimiento hipertextual. Por ejemplo:

-- el hipertexto no estructurado navegar un hipertexto

a. provoca problemas al

b. define funciones sobre cómo

c. contrarresta los efectos de

d. es habilitado al

Por último, la subescala de analogías requería que los estudiantes completaran 10 analogías compuestas por cuatro conceptos del hipertexto. Por ejemplo: 
-- acceder a la información :: índice :: integrar información : .......
a. enlaces
b. hipermapas
c. generador de ideas
d. acceso multi-usuario

Estas preguntas se utilizaban para evaluar la adquisición de conocimiento estructural. Para proporcionar unos estándares de la evaluación, tres autores e investigadores del campo del hipertexto acordaron las respuestas para cada una de las preguntas. Además de evaluar el conocimiento estructural, se desarrollaron 10 preguntas sobre el recuerdo de información de órden inferior

El hipertexto utilizado para todas las investigaciones fue la versión HyperCard (Jonassen, Roebuck y Wang, 1990) del libro Hypertext/Hypermedia (Jonassen, 1989a). Este hipertexto es un sistema de navegación que consta de 240 tarjetas y 1167 enlaces en tres pilas ${ }^{4}$ apoyado por capacidades de marcado de páginas y de anotación limitada. Todos los tratamientos de los estudios contenían enlaces referenciales integrados en las tarjetas. Algunos términos del texto se encontraban resaltados, permitiendo a los alumnos recorrer inmediatamente los enlaces. Los tratamientos variaban en términos de la clase de navegador utilizado por los alumnos y las formas en que representaban la información estructural. Cada uno de los 75 nodos conceptuales importantes contenía una tarjeta principal de 'términos relacionados', que era la primera tarjeta accesible al atravesar un enlace a ese nodo. En el grupo de control, esta tarjeta proporcionaba una lista de términos relacionados con el concepto examinado en ese momento. Esta lista proporcionaba enlaces a esos nodos, pero no información estructural sobre los propios nodos o los enlaces. Los tratamientos experimentales (descritos con mayor detalle más adelante) sustituyeron estas listas con navegadores gráficos o mantuvieron las listas, aunque manifestando abiertamente la naturaleza de cada enlace cuando estaba siendo atravesado. Éstos estaban diseñados para proporcionar o requerir generar al alumno información estructural explícita sobre los nodos y los enlaces del hipertexto.

Las investigaciones involucraron a profesores en un programa de educación para profesores, a los que se les encargó aprender una importante y novedosa tecnología educativa, el hipertexto, como tarea en un curso previo al servicio sobre tecnología educativa. De forma individual, los estudiantes interactuaron y estudiaron el hipertexto durante una o dos horas para adquirir tanta información sobre esta nueva tecnología como les fuera posible. Un programa de monitorización se añadió a las pilas para auditar las interacciones de los estudiantes.

\section{Estudio 1: Lista vs mapa vs ventana de enlaces}

\section{Cuestiones}

En el primer estudio hemos comparado la medida en que los usuarios adquieren conocimiento estructural al usar un navegador gráfico frente a la mediación de una ven-

$4 \quad$ (N. del T.) "Stacks" en el original. 
tana pop-up en cada enlace. Cada navegador gráfico mostró el nodo actual y todos los nodos enlazados directamente con él y describió la naturaleza del enlace entre cada uno de dichos nodos (véase la Figura 2 como ejemplo). Cada una de las cajas en el navegador gráfico hacía de botón de acceso rápido. Para navegar el hipertexto, los usuarios harían clic en cualquiera de las cajas para atravesar el enlace hacia ese tema.

En el segundo tratamiento experimental, los nodos relacionados con el nodo actual se representaban como una lista (sin mostrar información estructural). Para atravesar un enlace, los usuarios simplemente harían clic sobre un término. Una vez el usuario pulsara el término para seleccionarlo, es decir, para navegar por ese enlace, aparecería una ventana llenando el $70 \%$ de la pantalla. El texto de esta ventana emergente especificaba de forma explícita la naturaleza de la relación entre el nodo que el alumno estaba abandonando y el nodo al que se estaba dirigiendo. De esta forma, siempre que el estudiante atravesara un enlace entre los nodos existente y objetivo, la ventana establecía de forma explícita la relación entre el nodo que estaban abandonando y el que habían seleccionado, proporcionando una declaración explícita de conocimiento estructural.

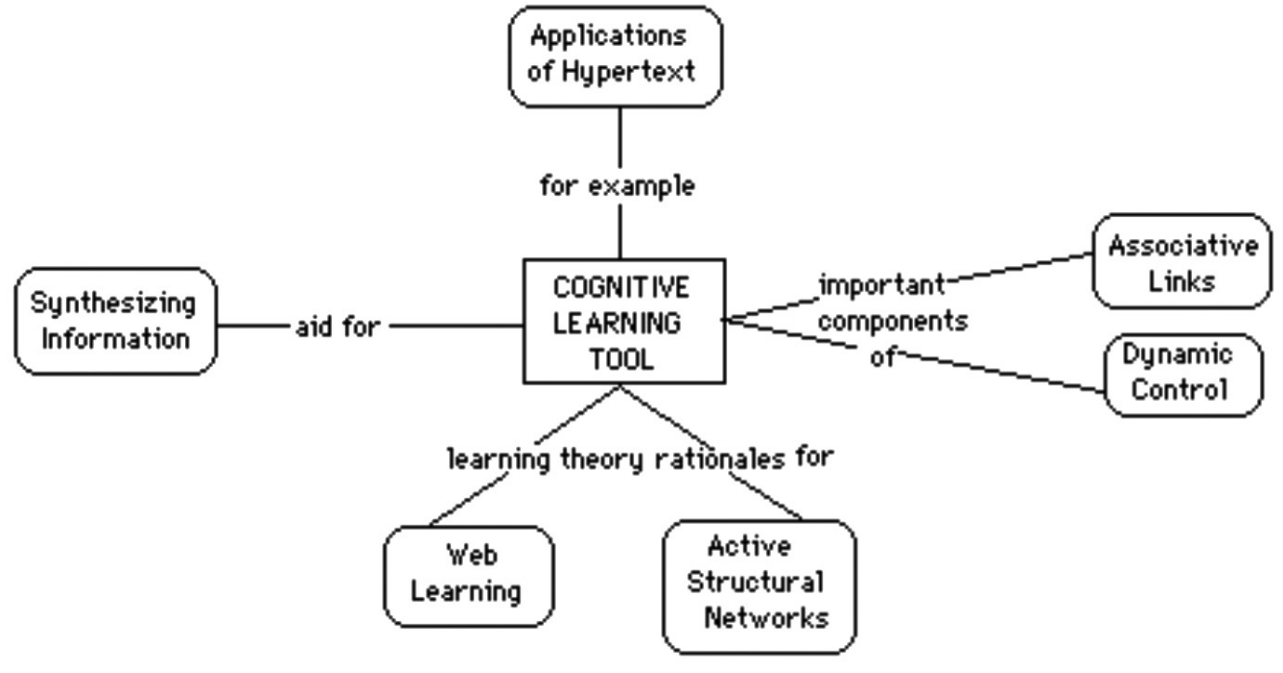

Figura 2. Navegador gráfico utilizado en el estudio

Ambas condiciones experimentales proporcionaban información estructural al estudiante. El tratamiento del navegador gráfico ilustraba la organización de la información en la base de conocimiento hipertextual, mientras que el tratamiento de ventanas emergentes establecía de forma explícita las relaciones mediante un criterio nodo-por-nodo.

\section{Resultados}

Las variables dependientes de este estudio incluían recuerdo, relación, proximidad y subescalas de analogía del post-test. Las subescalas de recuerdo (15 ítems), relación (10 ítems), y analogía (10 ítems) eran sumadas en cada uno de los temas. Las va- 
loraciones de proximidad se compararon con las valoraciones de los expertos. Para puntuaciones de proximidad, se calcularon coeficientes de correlación en cada uno de los temas, en los que las valoraciones del tema se correlacionaron con las valoraciones de proximidad de un grupo de tres expertos.

Se hizo un análisis de varianza para cada una de las cuatro variables dependientes. La única variable dependiente con una diferencia marginal significativa fue la variable de recuerdo $(\mathrm{F}(2,93)=2.75, \mathrm{p}<.07)$. Un análisis post hoc evidenció que las versiones de control y de mapa difirieron significativamente, con el grupo de control $(\mathrm{M}=7.8)$ superando a la versión de mapa $(\mathrm{M}=6.6)$, las puntuaciones más bajas a nivel global.

Las puntuaciones de las tareas de conocimiento estructural fueron esencialmente equivalentes. Las puntuaciones más altas en relación $(\mathrm{M}=5.6)$ y proximidad $(\mathrm{M}=.62)$ se contabilizaron en el tratamiento de ventanas emergentes, sin embargo esta diferencia no fue significativa. Puntuaciones análogas para los tres grupos resultaron vagamente superiores en este caso (a nivel global $\mathrm{M}=2.9$ ). La dificultad de procesamiento de una tarea de razonamiento de alto nivel como las analogías fue muy problemática para estos estudiantes.

Sin embargo, se encontraron evidencias de que los estudiantes estaban procesando parte de la información estructural. La cantidad total de tarjetas estructuralmente relacionadas a las que accedieron los estudiantes también fue calculada. Las tarjetas estructurales eran los mapas (en el grupo de mapas) o las listas del tratamiento de ventanas emergentes y del grupo de control. Estas tarjetas contenían la información estructural que definía las relaciones entre nodos en la base de conocimiento. El número de tarjetas estructurales accedidas fue distinto entre grupos $(\mathrm{F}(2,93)=3.75$, $\mathrm{p}<.05)$ mientras que el número total de tarjetas a las que se accedió y el tiempo total dedicado a la visualización de tarjetas estructurales no difirieron significativamente. El grupo de control accedió a 106 tarjetas, mientras que los grupos de ventanas emergentes y de mapas accedieron a 85 y 92 tarjetas respectivamente.

La ausencia de efectos para los resultados de conocimiento estructural no significa necesariamente que no aconteciera codificación estructural. Cuando el número de tarjetas de información estructural accedidas durante el estudio disminuyó en las variables dependientes, hubo diferencias significativas. El número de tarjetas estructurales de alto nivel (en el mapa principal y los ocho mapas de primer nivel, p. ej., Características del Hipertexto) a las que accedieron los estudiantes predijeron significativamente las puntuaciones de relación $(\mathrm{F}=3.7, \mathrm{p}=.05)$. El número de tarjetas estructurales a las que se accedió también anticipaba significativamente el tiempo empleado $(\mathrm{F}=5.37$, $\mathrm{p}<.05$ ). Así pues, acceder a información en el hipertexto sí que anticipaba la cantidad de conocimiento estructural adquirido. El tiempo total empleado en tarjetas estructurales también se calculaba por grupo. El tiempo empleado en tarjetas estructurales no anticipaba significativamente ninguna de las variables dependientes.

\section{Estudio 2: Procesamiento generativo de información estructural}

\section{Cuestiones}

Una de las debilidades del primer estudio era que no se proporcionaba soporte didáctico al estudiante. El hipertexto es una tecnología que ayuda a la recuperación de 
información y tareas de búsqueda. Sin embargo, estas funciones no están necesariamente correlacionadas con la enseñanza. Uno de los problemas potenciales más importantes en el aprendizaje desde el hipertexto es la integración de la información ofrecida en estructuras de conocimiento (Jonassen, 1989a). Aprender requiere que los usuarios no sólo accedan a la información sino que también la interpreten al relacionarla con el conocimiento previo.

Por tanto, en el segundo estudio hemos tratado de proporcionar soporte didáctico adicional incluyendo un tipo de tratamiento más generativo. El aprendizaje generativo sucede cuando los estudiantes relacionan de manera significativa información con conocimiento previo (Wittrock, 1974). El tratamiento de control se comparó con el tratamiento de ventanas emergentes (al igual que en el Estudio 1) y con el tratamiento de procesamiento generativo. El tratamiento generativo era similar en apariencia con la versión de ventanas emergentes del mismo hipertexto utilizado en el Estudio 1. Sin embargo, en lugar de informar sobre cuál era la naturaleza de la relación entre los nodos enlazados, se pedía a los estudiantes que clasificaran la naturaleza de cada enlace por ellos mismos. La ventana emergente presentaba 12 tipos de enlaces distintos y requerían que el estudiante determinara cuál de los tipos de enlace describía más acertadamente la naturaleza de la relación implicada por el enlace que estaban atravesando. El usuario tenía la opción de volver al nodo previo o avanzar para ver el nodo que habían seleccionado tantas veces como fuera necesario. Se proporcionaba información sobre los resultados para cada selección del usuario hasta que éste seleccionara el tipo de enlace correcto.

\section{Resultados}

Al igual que en el primer estudio, no se encontraron efectos significativos a nivel de .05. Como en el primer estudio, las puntuaciones de recuerdo difirieron $(\mathrm{F}=2.87$, $\mathrm{p}=.06)$. Un test post hoc evidenció que las puntuaciones del grupo de control $(\mathrm{M}=5.38)$ fueron significativamente mayores que las puntuaciones del grupo generativo (4.42). Como en el Estudio 1, los sujetos del grupo de control estuvieron mejor capacitados para recordar información, al estar menos distraídos por las actividades de conocimiento estructural. Las puntuaciones de relaciones fueron ligeramente más altas para ambos tratamientos experimentales $(M=5.75)$ que para el grupo de control $(M=5.1)$, pese a que esta diferencia no fue significativa. Las puntuaciones de analogía para los tres grupos apenas superaron lo fortuito $(\mathrm{M}=2.8$ en general) y no fueron significativas. Como en el primer estudio, estos estudiantes consideraron que las preguntas de analogía eran bastante difíciles. Como estaba previsto, las puntuaciones de recuerdo fueron superiores en el grupo de control, pero ninguna de las estrategias estructurales produjeron un incremento en la adquisición de conocimiento estructural.

\section{Estudio 3}

\section{Cuestiones}

Aunque las tareas didácticas normalmente se diseñan para servir como indicador importante de la integración de la información en las estructuras de conocimiento 
(pese a que esto no se contemplaba en los Estudios 1 y 2), probablemente el determinante más importante del aprendizaje es la conciencia y comprensión de la tarea requerida o resultado del aprendizaje. En los dos estudios previos, quedó claro que los estudiantes no tenían claro cómo ‘aprender del hipertexto' o cómo procesar información estructural. Los estudiantes de estos estudios estaban más familiarizados con el desempeño de tareas específicas, convergentes y orientadas al recuerdo. Se volvió obvia durante el curso de la experimentación la incertidumbre implicada en el intento de integrar conocimiento cuando la tarea no estaba clara y cuando la forma de comprender el material era tan diferente. Así pues, el tercer estudio proporcionó un enfoque en el conocimiento más deliberadamente estructural mediante el uso de la interconexión semántica como práctica y estrategia de evaluación. Es decir, este estudio obligaba a los estudiantes a crear una red semántica como estrategia de aprendizaje integrada, una tarea que requería la adquisición y manifestación de conocimiento estructural.

\section{La interconexión semántica como estrategia integradora}

El software para la interconexión semántica podría usarse como estrategia de aprendizaje cognitivo para ayudar a los estudiantes a integrar la información del hipertexto (Jonassen, 1989b). El proceso de navegar un hipertexto modela el proceso de acumulación del aprendizaje (Rumelhart y Norman, 1978). Sin embargo, un problema importante del aprendizaje a partir un hipertexto es la integración de aquello que se adquiere al navegar dentro de la estructura de conocimiento del estudiante (Jonassen, 1989b). Añadiendo un programa de interconexión semántica al navegador de hipertexto proporcionamos a los estudiantes una herramienta para integrar la información en su estructura de conocimiento. Este proceso de interconexión semántica permite el proceso de reestructuración definido por Rumelhart y Norman.

\section{Métodos}

El tercer estudio asignó a la mitad de los estudiantes la responsabilidad de construir una red semántica sobre el tema del hipertexto tras el período de estudio. A la otra mitad sólo se le pidió estudiar el hipertexto para adquirir conocimiento durante el período de estudio. En el tratamiento experimental, proporcionamos a los estudiantes el programa de interconexión semántica, Learning Tool ${ }^{5}$ (véase la Figura 1 como ejemplo) y se les informó que, tras navegar por el hipertexto, serían responsables de producir una red semántica sobre el tema del hipertexto. Estos estudiantes habían adquirido competencia con el programa de interconexión semántica previamente. Al igual que en los otros estudios se evaluaron los efectos sobre el aprendizaje inmediatamente después del tratamiento y antes de que cualquier red semántica fuera creada, de modo que el tiempo empleado en la tarea no difirió entre grupos. Nuestro propósito era centrar su atención en la información estructural con el conocimiento de que se les pediría producir una red semántica.

Este tercer estudio incluía un segundo factor, la clase de soporte al conocimiento estructural en la interfaz. Al tratamiento de control se le proporcionó la interfaz lista

(N. del T.) "Learning Tool”, nombre del programa, se traduciría como "Herramienta de Aprendizaje". 
(como en los Estudios 1 y 2) que no ofrecía señales estructurales. Al grupo experimental se le proporcionó un tratamiento de navegador gráfico, como en el primer estudio.

\section{Resultados}

En el tercer estudio, los estudiantes a los que se les dio la tarea de crear una red semántica obtuvieron resultados significativamente mejores $(M=6.6)$ en el área de interpretación respecto a los dos grupos a los que sólo se les pidió estudiar los materiales $(\mathrm{M}=5.2)$, de acuerdo con el análisis de varianza de dos factores, $\mathrm{f}=7.82$, $\mathrm{p}<0.1$. Este resultado indica que al centrar la atención del estudiante en aspectos estructurales de la información del hipertexto, se mejoró sustancialmente la adquisición de conocimiento estructural. El navegador gráfico ayudó en este proceso. Los estudiantes a los que se proporcionó navegadores gráficos puntuaron ligera pero no significativamente mejor que el grupo de control.

No se encontraron diferencias en las puntuaciones de recuerdo. Sin embargo, un análisis de varianza de un factor comparando las puntuaciones entre los cuatro grupos de tratamiento indicó que el grupo de navegador gráfico/interconexión semántica funcionó significativamente mejor en la subescala de analogía, $\mathrm{F}=2.77, \mathrm{p}<.05$. Parece que el soporte visual del navegador gráfico junto al foco sobre relaciones estructurales aumentaron las comparaciones necesarias para completar analogías.

\section{Conclusiones}

\section{Atención a la información estructural}

En la medida en que los estudiantes atendieron a las señales estructurales proporcionadas en los hipertextos, adquirieron algún conocimiento estructural. Los análisis de regresión post hoc evidenciaron que los estudiantes estaban, de hecho, atendiendo a parte de la información estructural. El número de tarjetas estructurales accedidas pareció predecir la adquisición de conocimiento estructural en la forma de juicios de relación en dos formas distintas. El tiempo empleado con las tarjetas estructurales sugirió una relación entre el acceso a dichas tarjetas y el nivel de adquisición de conocimiento estructural. Estos efectos son débiles en el mejor de los casos, pese a que una mayor exposición al hipertexto podría corroborar estos resultados.

De los resultados sólo podemos concluir, sin embargo, que el mero hecho de proporcionar señales estructurales en la interfaz de usuario de un hipertexto no resultará en un incremento significativo de la adquisición de conocimiento estructural. Que los sujetos del tratamiento de control recordaran más información es consistente con la investigación sobre la lectura que muestra que sin señalización o práctica, los estudiantes tienden a recordar microproposicones más fácilmente que macroproposiciones. Sin embargo, parece que la información estructural en la interfaz claramente obstaculizó el recuerdo de información por parte de los estudiantes en los tratamientos de conocimiento estructural. En cambio, no resultó en una adquisición de conocimiento estructural proporcionalmente mayor. Una explicación para la ausencia de adquisición de conocimiento estructural a partir de la señalización estructural en la 
interfaz es la "noción errónea en relación al hipertexto... de que las 'redes' arbitrarias de datos en sistemas de hipertexto tienen una gran importancia semántica" (Whalley, 1990, p.63), al menos para los estudiantes. Lo más importante en el aprendizaje es la construcción de una estructura de conocimiento personalmente relevante. Parece que la imposición arbitraria de redes semánticas podría no ser adecuada para sustituir a las personales, o al menos no para reproducirse directamente sobre las estructuras de conocimiento de los estudiantes. De modo que el mero hecho de mostrar a los estudiantes unas relaciones estructurales, sin un propósito para ello, probablemente no es suficiente para proporcionar una codificación profunda de dicha información. Cuando se requieren resultados de información estructural, los estudiantes aparentemente participan de la información estructural y la codifican en la memoria.

\section{Limitaciones cognitivas de la conducta de navegación}

Según Whalley (1990), el modo más natural de estudiar un hipertexto es la navegación. La pregunta es hasta qué punto la navegación libre puede mantener unos objetivos educativos, especialmente sin un propósito claramente establecido para el estudio. Los datos y comentarios de los estudiantes en los primeros dos estudios mostraron que carecían de un propósito claro para estudiar el hipertexto. Esa carencia de propósito se manifestó en el rendimiento uniforme entre grupos, especialmente con las tareas relacionadas con el conocimiento estructural. El aprendizaje desde hipertextos debe depender de tareas externamente impuestas o mediadas - simplemente navegar por una base de conocimiento no genera un procesamiento suficientemente profundo como para producir un aprendizaje relevante.

Los hipertextos obviamente son tecnologías de recuperación de información. Sin embargo, la recuperación de información no es suficiente por sí misma como para proporcionar un aprendizaje importante. Cuando los objetivos de acceso a la información requieren de un procesamiento más profundo, entonces es más probable que se produzca dicho procesamiento. No obstante, el simple navegar por el hipertexto no es motivación suficiente para proporcionar un aprendizaje relevante. Bien podría ser que el hipertexto no sea lo más apropiado para cometidos de aprendizaje altamente estructurados, tal como sugiere Duchastel (1990).

\section{Estrategias para el procesamiento de hipertextos}

Parece que los estudiantes en estas investigaciones, tal y como se esperaba, carecían de estrategias para el procesamiento hipertextual (Jonassen, 1989a), lo cual probablemente impidió un uso más eficaz de la tecnología. El hipertexto, y el mayor control formativo por parte del estudiante que comporta, es una forma novedosa de aprendizaje para estos estudiantes. Cuanto más novedosa es la apariencia del hipertexto (p. ej. la versión de navegador gráfico), más negativamente reaccionaron los estudiantes. Una evaluación justa del aprendizaje desde hipertextos sólo puede provenir de estudiantes instruidos en el ámbito del hipertexto que hayan desarrollado un conjunto de estrategias útiles para navegar e integrar información en forma de hipertexto. 


\section{Adquisición de conocimiento estructural}

La forma innovadora de instrucción, el hipertexto, fue solamente exacerbada por sus novedosas indicaciones estructurales y resultados para el conocimiento estructural. La provisión explícita de información estructural era algo inusual para estos estudiantes. El requisito para procesar dicha información e integrarla en sus propias estructuras de conocimiento resultó incluso más novedoso para ellos, lo cual afectó negativamente a su rendimiento en las tareas. La pobre ejecución por parte de los estudiantes en tareas de conocimiento estructural de alto nivel, especialmente las analogías, desvirtuaron la mayor parte de los efectos del tratamiento. Esto pone en duda la capacidad de los estudiantes para participar en un aprendizaje significativo frente a la simple recuperación de información del hipertexto, especialmente en el contexto de una tarea de aprendizaje.

\section{Agradecimientos}

Quisiera agradecer a Sherwood Wang por las contribuciones que ha hecho a la investigación documentada en este capítulo. Él es la otra mitad del 'nos' al que me he referido tan a menudo en el comentario de la investigación de este artículo. Él podría haber sido co-autor de este capítulo de no ser porque vivía a 5.000 millas de casa cuando fue escrito. También querría reconocer que este capítulo se escribió cuando era profesor visitante en la facultad de Tecnología de la Enseñanza de la Universidad de Twente en los Países Bajos. Mis más sinceros agradecimientos para ellos por su apoyo.

\section{Bibliografía}

Bush, V. (1945) As we may think. Atlantic Monthly, 176(1), 101-108.

Buzan, T. (1974) Use Both Sides of Your Brain. New York: Dutton.

Diekhoff, G. M. (1983) Testing through relationship judgements. Journal of Educational Psychology, 75, 227-233.

Duchastel, P. (1990) Formal and informal learning with hypermedia. In D. Jonassen and H. Mandl (eds.) Designing Hypermedia for Learning. Berlin: Springer-Verlag.

Fiderio, J. (1988) A grand vision. Byte, October, 237-243.

Fields, A. (1982) Pattern notes: How to get started. In D. H. Jonassen (ed.) The Technology of Text: Principles for Structuring, Designing, and Displaying Text, Volume 1. Englewood Cliffs, NJ: Educational Technology Publications.

Fisher, K. M., Faletti, J., Thronton, R. Patterson, H., Lipson, J., and Spring, C. (1988) Computer-based knowledge representation as a tool for students and teachers. Paper presented at the annual meeting of the American Educational Research Association, New Orleans, LA, April.

Jonassen, D. H. (1987) Assessing cognitive structure: Verifying a method using pattern notes. Journal of Research and Development in Education, 20(3), 1-14.

Jonassen, D. H. (1988) Designing structured hypertext and structuring access to hypertext. Educational Technology, 28(10), 13-16. 
Jonassen, D. H. (1989a) Hypertext/Hypermedia. Englewood Cliffs, NJ: Educational Technology Publications.

Jonassen, D. H. (1989b) Mapping the structure of content in instructional systems technology. Educational Technology, 29(4).

Jonassen, D. H. (1990) Semantic network elicitation: tools for structuring hypertext. In C. Green and R. McAleese (eds.) Hypertext: State of the Art. Oxford: Intellect.

Jonassen, D. H. (1991a) Hypertext as instructional design. Educational Technology: Research and Development, 39(1), 83-92.

Jonassen, D. H. (1991b) Representing the expert's knowledge in hypertext. Impact Assessment Bulletin, 9(1), 1-13.

Jonassen, D. H. and Mandl, H. (1990) (eds.) Designing Hypermedia for Learning. Berlin: Springer-Verlag.

Jonassen, D. H., Roebuck, N. and Wang, S. (1990) Hypertext/Hypermedia (HyperCard Version). Englewood Cliffs, NJ: Educational Technology Publications.

Jonassen, D. H. and Wang, S. (1990) Conveying structural knowledge in hypertext knowledge bases. Paper presented at the annual meeting of the Association for the Development of Computer-based Instructional Systems, San Diego, CA, October 30-31.

Jonassen, D. H. and Wang, S. (1991) Conveying structural knowledge in hypertext knowledge bases. Paper presented at the annual meeting of the Association for Educational Communications and Technology, Orlando, FL, February 14-17.

Kozma, R. B. (1987) The implications of cognitive psychology for computer-based learning tools. Educational Technology, 28(11), 20-25.

Lambiotte, J. G., Dansereau, D. F., Cross, D. R., and Reynolds, S. B. (1989) Multi- relational semantic maps. Educational Psychology Review, 1(4), 331-347.

McAleese, R. (1990) Concepts as hypertext nodes: The ability to learn while navigating through hypertext nets. In D. Jonassen and H. Mandl (eds.) Designing Hypermedia for Learning. Berlin: Springer-Verlag.

McDonald, J. E., Paap, K. R., and McDonald, D. R. (1990) Hypertext perspectives: Using Pathfinder to build hypertext systems. In R. W. Schvanenveldt (ed.) Pathfinder Associative Networks: Studies in Knowledge Organisation. Norwood, NJ: Ablex.

Norman, D. A. (1976) Studies in learning and self-contained education systems, 1973-1976. Tech Report No. 7601. Washington, DC: Office of Naval Research, Advanced Research Projects Agency. (ED 121 786)

Norman, D. A., Gentner, S. and Stevens, A. L. (1976) Comments on learning schemata and memory representation. In D. Klahr (ed.) Cognition and Instruction. Hillsdale, NJ: Lawrence Erlbaum Associates.

Preece, F. F. W. (1976) Mapping cognitive structure: A comparison of methods. Journal of Educational Psychology, 68, 1-8.

Rumelhart, D. E. and Norman, D. A. (1978) Accretion, tuning and restructuring: Three modes of learning. In J. W. Cotton and R. Klatzky (eds.) Semantic Factors in Cognition. Hillsdale, NJ: Lawrence Erlbaum Associates.

Rumelhart, D. and Ortony, A. (1977) The representation of knowledge in memory. In R. C. Anderson, R. J. Spiro and W. E. Montague (eds.) Schooling and the Acquisition of Knowledge. Hillsdale, NJ: Lawrence Erlbaum Associates.

Shavelson, R. (1974) Methods for examining representations of subject matter structure in students' memory. Journal of Research in Science Teaching, 11, 231-249. 
Thro, M. P. (1978) Individual differences among college students in cognitive structure and physics performance. Paper presented at the annual meeting of the American Educational Research Association, Toronto, Canada.

Whalley, P. (1990) Models of hypertext structure and learning. In D. Jonassen and H. Mandl (eds.) Designing Hypermedia for Learning. Berlin: Springer-Verlag.

Wittrock, M. C. (1974) Learning as a generative activity. Educational Psychologist, 11, 8795. 